\title{
Famílias com Trabalho e Famílias sem Trabalho: Evidências de Polarização para o Brasil
}

\author{
- LUIZ GUILHERME SCORZAFAVE*
}

\author{
- NAÉrCio AQUino MENEZES-FILHO**
}

\begin{abstract}
RESUMO
O presente artigo analisa a polarização do trabalho entre domicílios no Brasil. Definimos polarização como a diferença entre a proporção observada de domicílios sem nenhum adulto trabalhando e a proporção que seria esperada caso o trabalho fosse aleatoriamente distribuído na população. Calculamos uma série de medidas de polarização para o Brasil utilizando os dados da Pesquisa Nacional por Amostra de Domicílios (PNAD) para os anos de 198I a 2003. Os resultados mostram que no Brasil a polarização do trabalho é menor do que nos países desenvolvidos para os quais ela foi calculada. Por outro lado, houve crescimento da polarização no período, em decorrência do aumento da fração de domicílios em que os dois adultos presentes não trabalhavam.
\end{abstract}

Palavras-Chave

polarização, trabalho, Brasil, desemprego

\begin{abstract}
This article analyzes the work polarization among households in Brazil. We define polarization as the difference between the observed ratio of workless households and the ratio that would have prevailed had work been randomly assigned to people. We calculate a series of polarization measures using the data from the Pesquisa Nacional por Amostra de Domicilios (PNAD) between I98I and 2003. The results show that polarization in Brazil is smaller than in the developed countries. On the other hand, polarization has increased in the period, a result of a rise in the fraction of two-adults workless households.
\end{abstract}

\section{KEY WORDS}

polarization, work, Brazil, joblessness

\author{
JEL CLASSIFICATION \\ J60, C00
}

\footnotetext{
+ Os autores agradecem a Pedro Henrique Navarrete pela excelente assistência de pesquisa. Luiz Guilherme Scorzafave agradece ao CNPq pelos recursos disponibilizados no âmbito do Edital 6I/2005.

* Professor da FEA-RP/USP. Endereço para contato: Av. Bandeirantes, 3900 - FEA-RP/USP - Ribeirão Preto - SP. CEP: 14040-900. E-mail: scorza@usp.br.

** Professor do IBMEC/SP e FEA/USP. E-mail: naercioamf@isp.edu.br.

(Recebido em fevereiro de 2006. Aceito para publicação em janeiro de 2007).
} 


\section{INTRODUÇÃO}

Nos últimos anos, um grande número de trabalhos vem utilizando os dados da Pesquisa Nacional por Amostras de Domicílio (PNAD) e da Pesquisa Mensal de Emprego (PME) como fonte de informação para estudos sobre os mais diversos aspectos relacionados ao mercado de trabalho, como o desemprego, a inatividade, a distribuição de renda, entre outros. No que diz respeito ao desemprego e à inatividade, o cálculo da taxa agregada de incidência destes fenômenos é realizado com base na agregação dos dados individuais para a população ou para o grupo de interesse do estudo. Apesar desta profusão de estudos envolvendo os microdados para cálculo das taxas de desemprego e inatividade, ${ }^{1}$ praticamente nenhum esforço tem sido feito no sentido de tentar associar a evolução destes indicadores com a parcela de membros desempregados ou inativos dentro de cada unidade familiar. Particularmente, o estudo da chamada polarização do trabalho entre domić́lios - isto é, em que medida o trabalho está concentrado em determinados domicílios - não tem sido explorado no Brasil.

Cabe ressaltar que outros trabalhos já levaram em consideração os demais membros da família nas decisões referentes ao mercado de trabalho, embora nenhum deles aborde a questão dos domicílios sem trabalho e da polarização do trabalho entre domićlios. Há trabalhos para o Brasil que procuram captar o efeito trabalhador adicionado, como, por exemplo, a entrada da esposa em resposta ao desemprego do marido (Fernandes e Felício, 2005) e o efeito trabalhador desalentado, decorrente da desistência do indivíduo desempregado em continuar procurando trabalho (Gonzaga e Reis, 2005). Já no contexto da decisão de oferta de horas de trabalho, pode-se relacionar as condições dos outros membros do domicílio à oferta de trabalho feminina (Avelino e MenezesFilho, 2003) ou ao trabalho infantil (Kassouf, 2002).

Para compreendermos o conceito de polarização do trabalho, definimos a taxa de "não-trabalho" como a proporção de indivíduos inativos e desempregados na população total. Para uma noção mais precisa do que se trata e da importância desta questão, e seguindo Gregg, Scuttella e Wadsworth (2004, p. 6), "suponha um mundo simples onde haja apenas duas familias com dois membros cada e a taxa de não-trabalho na população seja de 50\%. O mundo em que um adulto de cada domicilio está sem trabalho é bem diferente daquele em que ambos trabalham em um domicilio e nenhum no outro. De fato, a taxa de não-trabalho agregada na população é a mesma, e portanto não consegue distinguir entre estes dois resultados.”

1 Ver, por exemplo, Picchetti e Menezes-Filho (2000, 2001), Picchetti e Fernandes (1999) e Chahad e Picchetti (2003). 
Mas, por que deveríamos nos preocupar com a concentração do "não-trabalho" em alguns domicílios? Um dos problemas, como apontam Gregg e Wadsworth (2002), é que domicílios nos quais ninguém trabalha têm maior probabilidade de serem dependentes de programas governamentais e de estarem em situação de pobreza. Além disso, se nestes domicílios morarem crianças, maior a chance destas se envolverem em trabalho infantil.

Vamos supor, ademais, que um país esteja passando por um processo de redução do desemprego agregado. Se os indivíduos que saem da situação de desemprego residem em domićlios em que já havia outros membros trabalhando, o efeito da redução do desemprego é menor do que se alguém de um domicílio totalmente desempregado passasse a trabalhar. Também é nítida a importância do estudo da polarização para avaliar o impacto das políticas públicas considerando-se a situação domiciliar dos envolvidos. Por exemplo, uma política de incentivo ao emprego juvenil pode até obter êxito; entretanto, os jovens que obtêm o emprego podem residir em domicílios em que outros membros já estão empregados, enquanto que pode haver jovens que não se beneficiam da medida e que moram em domicílios em que ninguém trabalha. Assim, a análise dos domicílios sem trabalho permitiria identificar se este fenômeno estaria ocorrendo e as causas que estão por trás do fato do jovem que mora num domicílio sem trabalho não conseguir trabalhar.

Portanto, dada a ausência de qualquer evidência para o Brasil a respeito da polarização do trabalho entre domicílios, o objetivo do artigo é calcular este indicador para o caso brasileiro. Ou seja, queremos saber em que medida o trabalho está concentrado em determinados domicílios, trazendo conseqüências importantes, por exemplo, para o delineamento de políticas públicas de combate à pobreza.

Deste modo, além desta introdução, o artigo possui mais três seções. Na seção seguinte, apresentaremos a metodologia de cálculo da polarização, com base nos trabalhos de Gregg e Wadsworth (2001, 2002) e Gregg, Scutella e Wadsworth (2004). Na seção 2 é aplicada a metodologia para o caso brasileiro no período compreendido entre 1981 e 2003. Por fim, a última seção é dedicada às conclusões do trabalho.

\section{METODOLOGLA}

\subsection{Medindo a Polarização}

A metodologia para o cálculo da polarização é originada dos trabalhos de Gregg e Wadsworth (2001, 2002) e Gregg, Scutella e Wadsworth (2004). Esta seção se baseia, 
fundamentalmente, nestes trabalhos. Segundo estes autores, é preciso construir uma medida que possibilite saber o tamanho da diferença entre a taxa de "não-trabalho", medida em termos individuais e a mesma taxa medida em termos domiciliares. Além disso, é importante que tal medida seja decomposta de forma a permitir a investigação das causas dessa discrepância.

Gregg e Wadsworth (2002) propõem a análise deste problema sob a ótica do seguinte contrafactual: qual seria a proporção de domicílios sem trabalho se este fosse aleatoriamente distribuído na população? Sob o contrafactual, todos os indivíduos teriam a mesma probabilidade de não estarem trabalhando, dada pela taxa agregada de "não-trabalho" da população, $n$. Assim, a taxa prevista de "não-trabalho" para um domicílio com $i$ adultos no tempo $t$ é dada por:

$$
p_{i t}=n_{t}^{i}
$$

Ou seja, a taxa prevista de não-trabalho domiciliar é o produto das probabilidades não condicionais de não-trabalho individuais (mesma para todos os indivíduos), em que o número de termos do produto é dado pelo número de adultos do domicílio. Vale ressaltar que esta taxa é um contrafactual. Se ignorarmos o subscrito para o tempo, a taxa prevista de "não-trabalho" agregada é a média ponderada destas taxas em que os pesos são dados pelas parcelas de cada tipo de domicílio na população, $s_{i}$, sendo $s_{i}$ definida pelo número de adultos residentes no domicílio.

$$
\hat{w}=\sum_{i} s_{i} p_{i}=\sum_{i} s_{i} n^{i}
$$

Definimos polarização do trabalho entre grupos como a diferença entre a taxa domiciliar de não-trabalho observada (w), ou seja, a proporção de domicílios em que todos os membros não possuem trabalho, e a taxa prevista $(\hat{w})$. Assim, a polarização permite avaliar "em que medida há mais (ou menos) domicilios sem trabalho do que seria previsto por uma distribuição aleatória do trabalho." (Gregg e Wadsworth, 2002, p. 6).

$$
\text { Polarização }=w-\hat{w}=\sum_{i} s_{i} w_{i}-\sum_{i} s_{i} n^{i}=\sum_{i} s_{i}\left(w_{i}-n^{i}\right)
$$

em que $w_{i}$ é a proporção de domicílios com $i$ adultos com nenhum deles trabalhando. A equação (3) fornece uma medida da diferença (em pontos porcentuais) entre as medidas de "não-trabalho" calculadas com base no domicílio e no indivíduo. Quanto maior a medida, maior a polarização. Cabe salientar que a medida pode ser calculada para domicílios com diferentes números de membros para investigarmos que tipos de domicílios experimentaram maior polarização. 
É importante salientar que a polarização observada cresce se houver uma redução do trabalho concentrada nos domicílios com um só adulto. Além disso, a polarização prevista cresce mais se houver aumento na proporção de domicílios com um só adulto do que em resposta a aumento na mesma proporção nos domicílios com mais de um adulto. Por fim, "a taxa de não-trabalho individual e a proporção de domicilios sem ninguém trabalbando não precisam se alterar à mesma taxa ou na mesma direção ao longo do tempo." (Gregg e Wadsworth, 2001, p. 791). Por exemplo, se a criação de empregos ocorrer em domicílios com mais de um adulto, a taxa de "não-trabalho" individual cairá, mas a proporção de domicílios sem ninguém trabalhando não cairá.

\subsection{Decomposição da Polarização}

Antes de apresentarmos a decomposição da polarização, discutiremos a decomposição na taxa de não-trabalho prevista ( $\hat{w})$. Segundo Gregg, Scutella e Wadsworth (2004) - daqui em diante GSW (2004) - a evolução nesta taxa pode ser assim decomposta:

$\Delta \hat{w}=\Sigma_{i} \Delta s_{i t} n^{i t}=\Sigma_{i} \Delta s_{i} *\left[0,5\left(n^{i t}+n^{i t+1}\right)\right]+\sum_{i} \Delta n^{i t} *\left[0,5\left(s_{i}^{t}+s_{i}^{t+1}\right)\right]$

em que o primeiro termo capta o impacto de mudanças na taxa de não-trabalho observada ao longo do tempo e o segundo captura o efeito da mudança na composição domiciliar.

Com o objetivo de entender as causas da variação da polarização, Gregg e Wadsworth (2002) propõem a decomposição ao longo do tempo da polarização por meio da seguinte medida:

$\left(w^{t+1}-\hat{w}^{t+1}\right)-\left(w^{t}-\hat{w}^{t}\right)=\Delta(w-\hat{w})=\Sigma_{i} \Delta s_{i}\left(w_{i}-n^{i}\right)=$

$=\Sigma_{i} \Delta s_{i} *\left[0,5\left(w_{i}-n^{i}\right)^{t}+0,5\left(w_{i}-n^{i}\right)^{t+1}\right]+\Sigma_{i} \Delta\left(w_{i}-n^{i}\right) *\left[0,5 s_{i}^{t}+0,5 s_{i}^{t+1}\right]$

em que o primeiro termo fornece a contribuição da alteração nos tipos de domicílios (em termos de número de adultos), mantendo a polarização constante no valor médio dos dois períodos (entre grupos), e o segundo termo mede a mudança ocorrida dentro dos domicílios, mantendo-se a composição dos tipos de domicílios constante no valor médio dos dois períodos (dentro dos grupos).

Assim, segundo Gregg e Wadsworth (2001), o primeiro termo indica, por exemplo, se um aumento da polarização ocorre devido a aumento na proporção de domicílios com menores chances de ter todos os membros empregados, enquanto que o segundo termo fornece a contribuição de uma piora das condições gerais de emprego para todos os tipos de domicílios. O segundo termo pode ser novamente decomposto para 
se saber se a magnitude deste fator é mais forte em domicílios com um adulto ou em domicílios com mais de um adulto.

\subsection{Desagregação por Características}

Até o momento estamos utilizando a taxa de "não-trabalho" agregada para toda a população, $n$. No entanto, como apontam Gregg e Wadsworth (2001), podemos usar medidas de "não-trabalho" para subgrupos da população definidos de acordo com sexo, idade, região de residência e escolaridade. Desta forma, podemos saber se "membros de domicilios sem trabalho possuem características em comum que os torna mais propensos a estarem nesta situação. Por exemplo, se eles são mais velhos, menos qualificados ou vivem em regiões de elevado desemprego." (Gregg e Wadsworth, 2001, p. 792). É claro que quanto maior for o grau de desagregação dos grupos, maior será a convergência entre a taxa prevista e a observada.

Assim, definimos, seguindo GSW (2004), a taxa média de não-trabalho dos subgrupos desagregados de acordo com o vetor $K$ de características observáveis:

$$
E\left(n_{i} / K\right)=n_{k}
$$

Por exemplo, é possível que a taxa de não-trabalho seja diferente para indivíduos das grandes regióes do Brasil. Neste caso, $K$ corresponderia apenas à variável região. Se quisermos diferenciar as taxas de não trabalho por região e sexo, como, por exemplo, calcular a taxa de não-trabalho das mulheres na região Sul, $K$ conteria os atributos região e sexo.

Definimos, também com base em GSW (2004), a taxa domiciliar de não-trabalho prevista de cada domicílio como:

$$
E\left(w_{h}{ }^{K} / i, n, K\right)=\prod_{i} n_{k i}=w_{k i}
$$

Novamente, esta taxa é um contrafactual. Isto é, qual seria a taxa domiciliar de nãotrabalho se o mesmo fosse distribuído aleatoriamente entre os indivíduos de acordo com seu vetor de características $K$. Segundo Gregg, Scutella e Wadsworth (2004, p. 10), "este procedimento auxilia a entendermos se a polarização surge devido ao fato de que (i) domicílios com mais de um adulto têm características comuns nas quais o emprego varia significativamente ou (ii) se domicilios com apenas um adulto têm características que estão associadas com baixa probabilidade de emprego."

Com as duas expressões acima, podemos definir a polarização condicional como: 


$$
P\left(w, s_{i}, n, K\right)=w-\hat{w}_{k}=\sum_{i} s_{i} w_{i}-\sum_{i} s_{i} n_{k}^{i}=\sum_{i} s_{i}\left(w_{i}-n_{k}^{i}\right)
$$

Ou seja, de forma análoga à equação (3), a polarização condicional é dada pela diferença entre a proporção de domicílios sem trabalho e a taxa prevista pelo contrafactual, ponderada pela fração de domicílios de acordo com o número de adultos residentes nos mesmos. Cabe ressaltar que a polarização condicional permite uma interpretação mais precisa da taxa de não-trabalho individual como a probabilidade do indivíduo estar sem trabalho, já que leva em consideração que grupos de indivíduos com diferentes atributos teriam diferentes taxas de não-trabalho e, portanto, diferentes probabilidades de estar sem trabalho.

Gregg e Wadsworth (2001, p. 793) afirmam que divergências entre (3) e (8) podem ocorrer "porque todos os membros de certos domicílios possuem pouco acesso ao trabalho em comparação a outros domicílios com características similares."

\subsection{Dados}

A amostra utilizada neste estudo foi construída com base na Pesquisa Nacional por Amostra de Domicílios (PNAD) para os anos de 1981, 1985, 1989, 1993, 1997, 2001 e 2003. ${ }^{2}$ Foram excluídos da amostra os domicílios em que todos os adultos eram inativos e que a única renda era de aposentadoria ou pensão. Para o cálculo das medidas de não-trabalho e polarização foram considerados apenas os indivíduos com idade entre 18 e 60 anos. Foram excluídos os domicílios em área rural, já que a possibilidade de produção para consumo próprio é bem maior nestas áreas.

Além disso, a unidade de análise escolhida foi o domicílio em vez da família, já que é possível haver uma inter-relação entre famílias diferentes em um mesmo domicílio. Assim, por exemplo, um domicílio com duas famílias, sendo que em uma delas ninguém trabalha, provavelmente deve receber algum auxílio da outra família. Por sua vez, se ninguém de ambas as famílias trabalha, a situação é claramente pior.

\section{RESULTADOS}

Antes de discutirmos os resultados das medidas de polarização, vamos apresentar a taxa de não-trabalho da população urbana brasileira, a proporção de domicílios em

2 Devido a mudanças metodológicas a partir de 1992, os conceitos de ocupação e informalidade foram redefinidos a partir desse ano a fim de permitir a comparação com os dados até 1990. Para mais detalhes sobre a mudança metodológica, ver Guerra (1997). 
que ninguém trabalha e a fração de domicílios em que todos trabalham, por meio da Tabela 1.

TABELA I - TAXA DE NÂO-TRABALHO, PROPORÇÃO DE DOMICÍLIOS EM QUE TODOS TRABALHAM E PROPORÇÃO DE DOMICÍLIOS EM QUE NINGUÉM TRABALHA - 1981-2003 (EM \%)

\begin{tabular}{lcc}
\hline & 1981 & 2003 \\
\hline Domicílios em que todos trabalham & 29,7 & 43,9 \\
Domicílios em que ninguém trabalha & 4,9 & 6,9 \\
Taxa de não-trabalho & 38,0 & 34,1 \\
Inativos & $33,8(88,4 \%)$ & $25,8(75,0 \%)$ \\
Desempregados & $4,2(11,6 \%)$ & $8,3(25,0 \%)$ \\
\hline
\end{tabular}

Obs: A taxa de não trabalho se refere à proporção de pessoas que não trabalhavam.

Fonte: Elaboração própria com base nos dados da PNAD.

Os resultados apontam para um aumento expressivo da proporção de domicílios em que todos os adultos trabalham, no Brasil, entre 1981 e 2003. Este resultado está ligado à entrada das mulheres no mercado de trabalho, principalmente as cônjuges, conforme apontado por Scorzafave e Menezes-Filho (2001). Outros fatores que podem explicar este processo são: a redução do tamanho das famílias, o envelhecimento populacional e o aumento do nível educacional. Apesar deste incremento, o Brasil continuava, em 2003, com o menor porcentual de domicílios em que todos os adultos trabalham, se comparado à Grã-Bretanha $(65,5 \%)$, Estados Unidos $(66,0 \%)$, Alemanha $(62,4 \%)$ e Austrália $(61,7 \%){ }^{3}$ Apenas a Espanha apresenta indicadores mais baixos, com 20,9\% em 1984 e 38,9\% em 2000. Cabe salientar, entretanto, que em todos os países também houve crescimento neste indicador.

O Gráfico 1 permite uma melhor visualização desta tendência, apontando para um crescimento contínuo na proporção de domicílios em que todos os adultos trabalhavam até 1993 com posterior estabilização. No caso dos domicílios em que ninguém trabalha, há queda até 1989, com crescimento até 2003. Deste modo, estaria havendo um aumento da polarização do trabalho no Brasil, neste período.

3 Dados de 2000. Para mais detalhes, ver Gregg, Scutella e Wadsworth (2004). 


\section{GRÁFICO 1 - PROPORÇÃO DE DOMICÍLIOS EM QUE NINGUÉM TRABALHA E EM QUE TODOS TRABALHAM - 1981-2003}

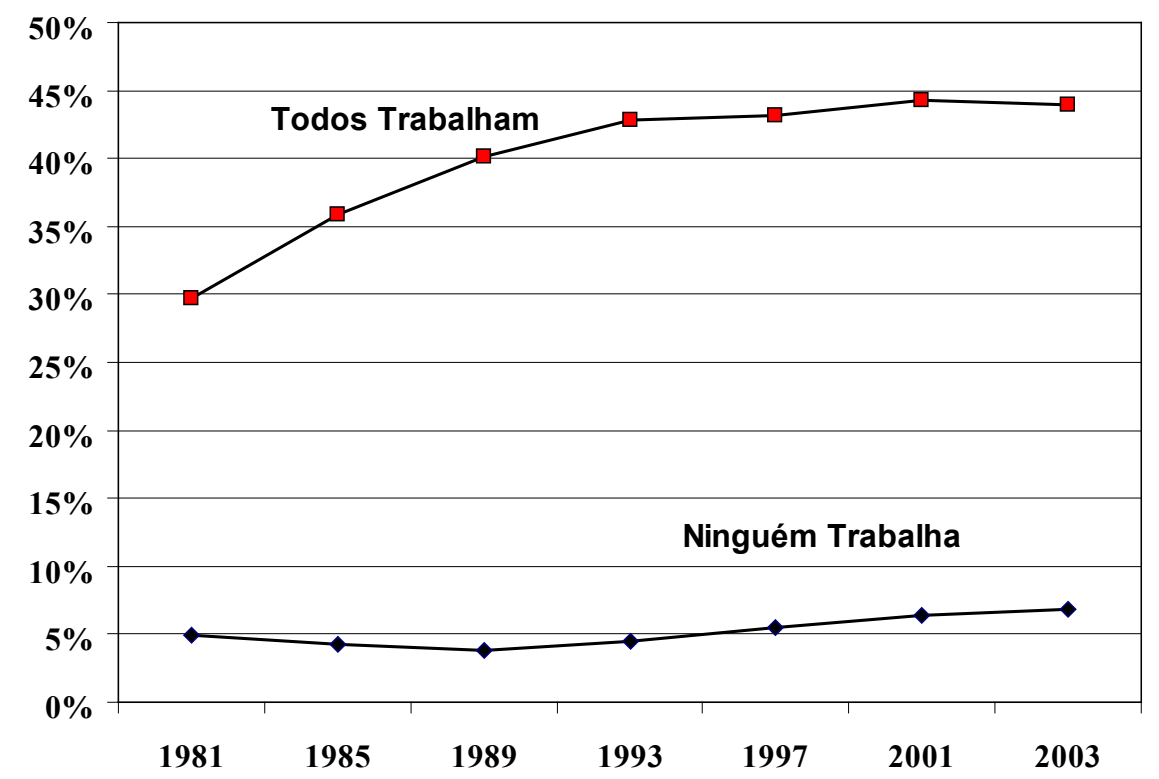

Fonte: Elaboração própria com base nos dados da PNAD.

No que diz respeito à proporção de domicílios sem trabalho, os números, para o Brasil, são menores que os apontados por GSW (2004). ${ }^{4}$ Porém, em todos os países, exceto os Estados Unidos, houve crescimento na fração de domicílios sem trabalho, assim como no caso brasileiro. Por fim, no que diz respeito à taxa de não-trabalho individual, há redução no Brasil e nos países estudados por GSW (2004). Portanto, de modo geral, o Brasil vem percebendo as mesmas tendências nestes três indicadores que as apontadas em GSW (2004).

Ainda com base na Tabela 1 , é interessante notar que dentre os não trabalhadores, os inativos perderam espaço relativamente aos desempregados, pois em 2003 estes últimos eram cerca de $25 \%$ dos não trabalhadores, comparativamente a $11,6 \%$ em 1981. Ou seja, o desemprego substituiu a inatividade na composição do "não-trabalho". Este fenômeno se deve a dois fatores: o aumento generalizado do desemprego e o aumento da taxa de atividade entre as mulheres, como pode ser visto na Tabela 2.

4 Em todos os casos, a taxa de não-trabalho variava de 11,6\% a 15,1\% em 1984 e de $10,7 \%$ a $16,9 \% \mathrm{em}$ 2000. Para mais detalhes, ver GSW (2004). 
TABELA 2 - TAXA DE DESEMPREGO E DE ATIVIDADE - HOMENS E MULHERES - 1981/2003 (EM \%)

\begin{tabular}{ccc}
\hline & 1981 & 2003 \\
\hline Taxa de Desemprego - Total & 6,6 & 11,4 \\
Homens & 5,6 & 9,0 \\
Mulheres & 8,6 & 14,5 \\
\hline Taxa de Atividade - Total & 66,4 & 74,4 \\
Homens & 91,0 & 87,9 \\
Mulheres & 42,8 & 62,0 \\
\hline
\end{tabular}

Fonte: Elaboração própria com base nos dados da PNAD.

Segundo GSW (2004), quanto maior o número de adultos presentes no domicílio, menor seria a probabilidade de que nenhum deles estivesse trabalhando. Assim, verificar a evolução da estrutura domiciliar e da proporção de domicílios sem trabalho de acordo com o número de adultos no domicílio, análise feita na Tabela 3, será de fundamental importância quando decompormos a mudança da taxa de não-trabalho domiciliar entre 1981 e 2003.

TABELA 3 - DOMICÍLIOS POR NÚMERO DE ADULTOS, FRAÇÃO DE DOMICÍLIOS EM QUE TODOS OS ADULTOS TRABALHAM E EM QUE NENHUM ADULTO TRABALHA - 1981/2003 (EM \%)

\begin{tabular}{lrcc}
\hline Adultos & Proporção & Ninguém trabalha & Todos trabalham \\
\hline 1981 & & & \\
1 & 8,8 & 12,9 & 87,1 \\
2 & 58,4 & 4,9 & 29,2 \\
3 & 16,8 & 4,1 & 20,0 \\
4 ou mais & 16,0 & 1,4 & 9,8 \\
\hline $\mathbf{2 0 0 3}$ & & & \\
1 & 13,8 & 15,4 & 84,6 \\
2 & 56,1 & 6,4 & 46,1 \\
3 & 17,3 & 4,8 & 25,4 \\
4 ou mais & 12,8 & 2,5 & 15,0 \\
\hline
\end{tabular}

Fonte: Elaboração própria com base nos dados da PNAD.

De fato, quanto maior o número de adultos no domicílio, menor é a chance de nenhum deles trabalhar. Além disso, a maior parte dos domicílios possui dois adultos, apesar do crescimento verificado naqueles com apenas um adulto. Esta tendência também se verifica na Austrália, Alemanha, Grã Bretanha e Espanha. (GSW, 2004). 
Outro ponto que chama a atenção é a estabilidade na fração de domicílios com três adultos.

No que diz respeito aos domicílios sem trabalho, houve crescimento entre todas as categorias de domicílio. Entretanto, em todos os grupos as taxas brasileiras são menores, em ambos os anos, que as verificadas nos países supracitados, exceto os Estados Unidos, que apresentou em 2000 taxas um pouco inferiores às brasileiras para domicílios com 2 adultos $(6,0 \%)$.

Por fim, vale destacar o forte incremento na proporção de domicílios em que os dois adultos presentes trabalhavam e aumentos menores para domicílios com três ou mais adultos. Deste modo, parece que outros membros da família, em geral, e as mulheres casadas, em particular, têm contribuído cada vez mais na geração da renda domiciliar por meio do seu forte ingresso no mercado de trabalho.

A seguir, apresentamos a medida de polarização para o caso brasileiro, calculada com base na equação (3).

TABELA 4 - MEDIDA DE POLARIZAÇ $\tilde{A O}-1981-2003$

\begin{tabular}{lcc}
\hline & 1981 & 2003 \\
\hline Taxa domiciliar de não-trabalho observada $(\mathrm{w})$ & 4,93 & 6,86 \\
Taxa domiciliar de não-trabalho prevista $(\hat{\mathrm{w}})$ & 12,94 & 12,03 \\
Polarização Simples (w - $\hat{\text { ŵ) }}$ & $-8,01$ & $-5,17$ \\
Taxa de não-trabalho individual $(\mathrm{n})$ & 38,0 & 34,1 \\
\hline
\end{tabular}

Fonte: Elaboração própria com base nos dados da PNAD.

O primeiro fato que chama a atenção na Tabela 4 é que a medida de polarização é negativa em ambos os períodos, no Brasil, embora tenha havido um aumento expressivo em 2003, mesmo quando se analisa a medida normalizada. A interpretação deste resultado é que no Brasil há menos domicílios sem trabalho do que seria esperado caso o trabalho fosse distribuído uniformemente entre os domicílios. Neste sentido, vale ressaltar que o valor dele para o Brasil é menor do que na maioria dos países analisados por GSW (2004). Por exemplo, no ano 2000, no caso da Grã-Bretanha, o índice foi de 6,1; na Austrália, 4,8; nos Estados Unidos, 1,0; na Alemanha, 0,4. Por sua vez, os resultados brasileiros, embora menores, se assemelham mais aos da Espanha

No que diz respeito à evolução da polarização, o Brasil caminhou na mesma direção dos países estudados por GSW (2004), apresentando crescimento do índice (3 pontos porcentuais). No caso da polarização simples, o crescimento foi de cerca de 3 pontos porcentuais. A situação brasileira se assemelha mais à da Espanha, que apesar do cres- 
cimento era, ainda em 2000, o único país estudado por GSW (2004) com polarização negativa. Em 1984, o índice espanhol era de -6,2, subindo para -2,1 em 2000. Por sua vez, a Grã-Bretanha apresentou crescimento de 6,3 pontos porcentuais, a partir do índice verificado em $1977(-0,2)$. Já a Austrália apresentou crescimento de 3,6 p.p. entre 1982 e 2000, enquanto os Estados Unidos teve crescimento de 2,1 p.p. entre 1977 e 2000. Por sua vez, a Alemanha experimentou crescimento de apenas 1,5 p.p. entre 1984 e 2000.

O Gráfico 2 indica que o aumento da polarização do trabalho no Brasil ocorreu de modo contínuo até 2001, quando, a partir de então, parou de crescer, ficando em $2003 \mathrm{em}$ torno de -5 pontos porcentuais. ${ }^{5}$

GRÁFICO 2 - EVOLUÇÃO DA POLARIZAÇÃO DO TRABALHO - 1981-2003

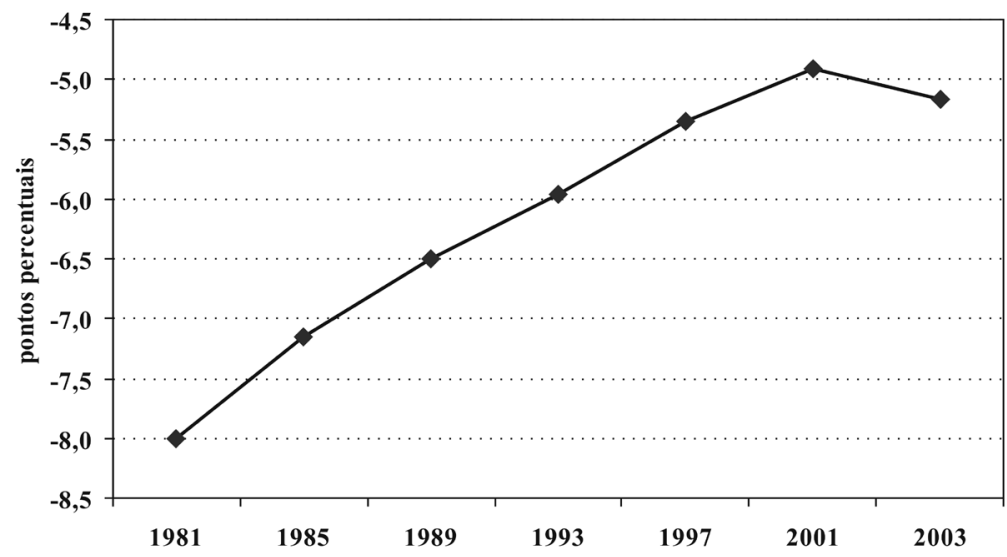

Fonte: Elaboração própria com base nos dados da PNAD.

Como GSW (2004, p. 12) apontam, uma redução da taxa de não-trabalho individual "deveria levar a um menor número de domicilios sem trabalho, enquanto as alterações na estrutura domiciliar em direção a domicilios com apenas um adulto deveriam gerar mais domicilios sem trabalho, tudo o mais constante." Por exemplo, com a redução da taxa de não-trabalho, se a distribuição de domicílios por número de adultos não mudar, é menor a probabilidade de se encontrar domicílios sem trabalho, já que alguma parte deste incremento no número de trabalhadores deve estar em domicílios nos quais antes ninguém trabalhava. Apesar de teoricamente ser possível que todo o aumento do emprego se concentre em domicílios em que já havia alguém trabalhando, isto é

5 Podemos dizer que quando o valor do índice de polarização é negativo, haveria subpolarização. Deste modo, um aumento no índice, a partir de valores negativos, caracterizaria uma redução da subpolarização. 
improvável. Como apontado nas Tabelas 1 e 3 , a taxa de não-trabalho brasileira caiu de $38,0 \%$ para $34,1 \%$, enquanto os domicílios com apenas um adulto passaram de $8,8 \%$ para $13,8 \%$ entre 1981 e 2003. Assim, é de fundamental importância identificar a responsabilidade de cada um destes fatores na evolução da polarização do trabalho. Com este intuito, a Tabela 5 e a Figura 1 apresentam o resultado das decomposições, realizadas com base nas equações (4) e (5).

\section{TABELA 5 - DECOMPOSIÇÃO DA POLARIZAÇÃO E DA TAXA DE NÃO- TRABALHO - 1981-2003}

\begin{tabular}{lc}
\hline Variação na taxa de não-trabalho domiciliar prevista & $-0,90$ \\
Impacto devido a mudança da composição domiciliar & 1,49 \\
Impacto devido a mudança na taxa de não-trabalho & $-2,39$ \\
\hline Variação na polarização & 2,84 \\
Decomposição entre tipos de domicílios & $-0,95$ \\
Decomposição dentro dos tipos de domicílios & 3,79 \\
\hline
\end{tabular}

Fonte: Elaboração própria com base nos dados da PNAD.

A primeira linha da Tabela 5 apresenta a alteração da taxa de não-trabalho domiciliar prevista entre 1981 e 2003. A redução da taxa de não-trabalho individual entre 1981 e 2003 contribui para reduzir a fração de domicílios sem trabalho em 2,4 pontos porcentuais, enquanto a alteração da estrutura domiciliar contribuiu para o aumento de 1,5 ponto porcentual na proporção de domicílios sem trabalho. Como o primeiro efeito é de maior magnitude, a taxa prevista de não-trabalho domiciliar foi menor em 2003 do que em 1981. Vale dizer que este resultado é semelhante ao obtido por GSW (2004): efeito trabalho negativo e efeito composição positivo. Entretanto, dependendo do país, o efeito líquido na taxa de não-trabalho prevista é diferente: positivo para a Grã-Bretanha, Alemanha e Espanha entre 1982 e 2000 e negativo para Austrália e Estados Unidos, similar ao resultado para o Brasil.

Na decomposição da variação da polarização, a alteração da distribuição de domicílios de acordo com o número de adultos contribui para reduzir o índice de polarização. Entretanto, este efeito é mais do que compensado pela parcela correspondente a alterações ocorridas dentro de cada tipo de domicílio. Ou seja, a alteração de $\left(w_{i}-n^{i}\right)$, dentro de cada tipo de domicílio $i$, explica todo o aumento da polarização.

A Figura 1 mostra que mais de $85 \%$ de todo o aumento da polarização ocorreram em domicílios com dois adultos, resultado em linha com os encontrados por GSW (2004) para Espanha (80\%), Estados Unidos (90\%), Alemanha (106\%) e Austrália (64\%), a exceção ficando por conta da Grã-Bretanha (30\%), em que a maior contribuição veio dos domicílios com um adulto. 
FIGURA I - ESQUEMA DA DECOMPOSIÇÃO DA POLARIZAÇÃO E DA TAXA DE NÃO-TRABALHO DOMICILIAR

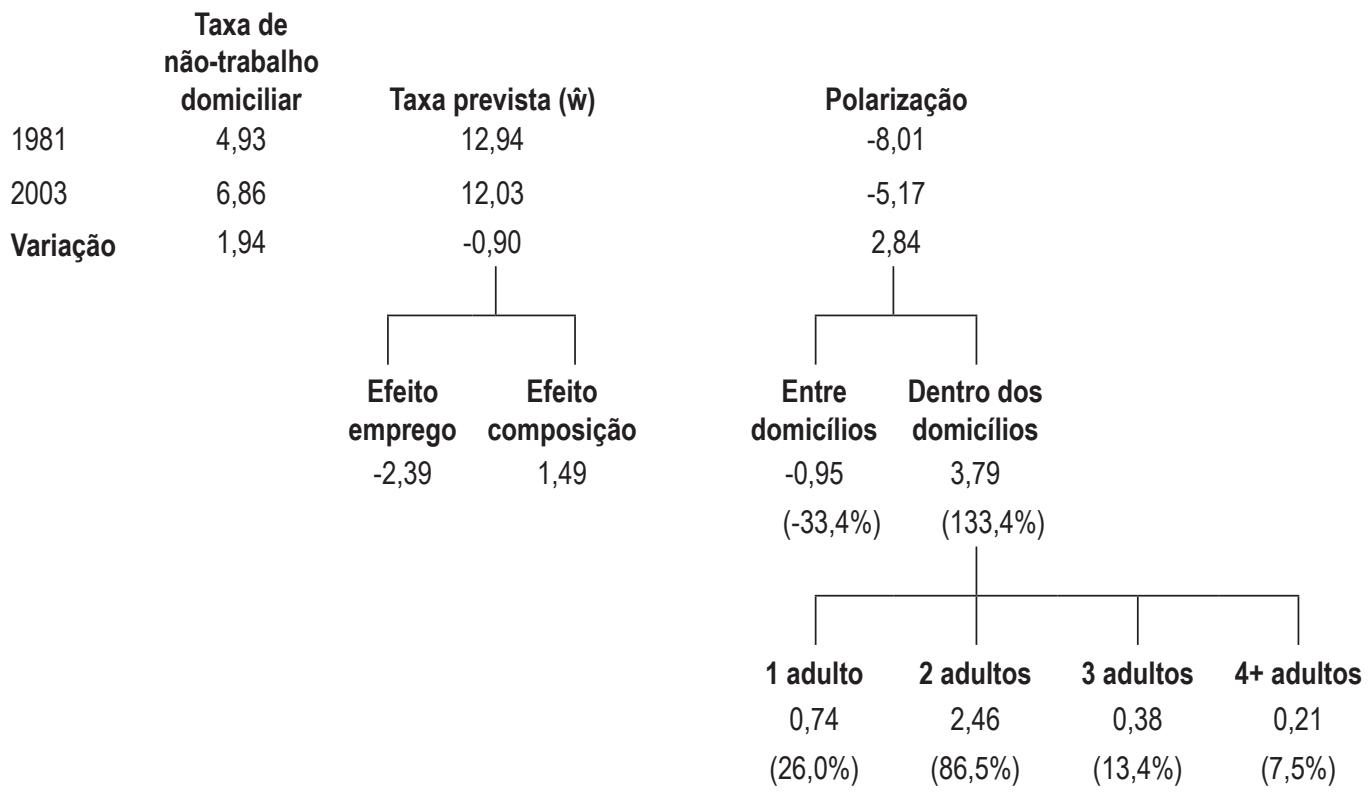

Fonte: Elaboração própria com base nos dados da PNAD.

A análise feita até aqui considerava uma medida de taxa de não-emprego individual que era a mesma, independente das características dos adultos do domicílio. Obviamente, as pessoas têm diferentes probabilidades de não estar trabalhando, dadas suas características observáveis. Assim, a Tabela 6 apresenta os valores da taxa de não-trabalho domiciliar condicional, obtidos com o cálculo da taxa de não-trabalho para k grupos diferentes. Seguindo Gregg e Wadsworth (2002), os atributos escolhidos foram idade, educação, região e sexo. Assim, os indivíduos foram divididos nos seguintes grupos: ${ }^{6}$

- 3 grupos etários: 18 a 32 anos; 33 a 45 anos; 46 a 64 anos;

- 4 grupos educacionais: 0 a 3; 4 a 7; 8 a 10; 11 ou mais anos de estudo;

- 5 regióes: Sul; Sudeste; Nordeste; Norte; Centro-Oeste;

- sexo.

6 A divisão dos atributos idade e educação foi feita de modo a garantir a representatividade das células. A única célula com menos de 100 observações possuía 84 indivíduos. 
TABELA 6 - TAXAS DE NÃO-TR ABALHO CONDICIONAIS - 1981-2003

\begin{tabular}{lrrr}
\hline & 1981 & 2003 & Variação \\
\hline Taxa domiciliar de não-trabalho observada $(\mathrm{w})$ & 4,93 & 6,86 & 1,94 \\
Taxa domiciliar de não-trabalho prevista não-condicional $(\hat{\mathrm{w}})$ & 12,94 & 12,03 & $-0,90$ \\
\hline Taxa domiciliar de não-trabalho prevista condicional $\left(\hat{\mathrm{w}}_{\mathrm{k}}\right)$ & & & \\
$\quad$ Sexo & 10,29 & 11,71 & 1,42 \\
$\quad$ Região & 13,50 & 12,47 & $-1,04$ \\
$\quad$ Idade & 13,15 & 12,03 & $-1,13$ \\
$\quad$ Educação & 13,76 & 12,71 & $-1,05$ \\
$\quad$ Sexo, Região, Idade e Educação combinados & 9,74 & 11,35 & 1,61 \\
\hline
\end{tabular}

Fonte: Elaboração própria com base nos dados da PNAD.

Quando levamos em consideração a variação do não-trabalho por sexo, notamos que há uma redução da taxa domiciliar prevista em 1981, comparada com a não-condicional. Entretanto, isto se verifica em menor grau em 2003. Uma explicação para este fato é que em 1981 menos mulheres trabalhavam, enquanto o emprego masculino já era elevado. Assim, havia grande diferença nas taxas estimadas de não-trabalho dos dois grupos $\left(\mathrm{n}_{\mathrm{k}}\right)$, levando a uma queda no valor previsto da taxa de não-trabalho domiciliar. À medida que houve um aumento do trabalho feminino, as diferenças de emprego entre homens e mulheres diminuíram, fazendo com que as taxas de nãotrabalho individual entre os sexos diferissem pouco em relação à taxa não-condicional. Como GSW (2004, p. 15) argumentam,

"converging employment patterns by gender changes the predicted distribution of work, generating more households where both individuals work and others where no-one works. This strongly suggests a declining gender specific division of labour, at least as far as participation in the workforce, hours may still differ substantially.”

Vale notar que resultado semelhante a este é obtido por GSW (2004) para diversos países, e por Dawkins, Gregg e Scutella (2002) para a Austrália. A consideração dos demais fatores faz com que as taxas condicionais pouco difiram da não-condicional. Aliás, a taxa de não-trabalho domiciliar prevista seria ainda maior se considerássemos a variação da taxa de não-trabalho por região, educação e idade separadamente. $\mathrm{O}$ caso da educação é o mais marcante deste fato, já que levando em conta as diferenças neste atributo, a taxa prevista de não-trabalho domiciliar subiria para $13,76 \% \mathrm{em} 1981 \mathrm{e}$ $12,71 \%$ em 2003.

Por fim, permitindo a variação de todas as características simultaneamente, obtivemos uma aproximação maior em relação às taxas de não-trabalho observadas em $1981 \mathrm{e}$ 
2003, embora a diferença em relação à taxa não-condicional não seja tão grande. De forma geral, porém, a maior parte da polarização permanece inexplicada, mesmo levando em consideração a análise condicional em todas as características.

Como vimos anteriormente, a maior variação da polarização se deu dentro dos tipos de domicílios e não devido à alteração do tamanho relativo dos mesmos. Assim, iremos agora analisar mais detalhadamente as medidas de taxa de não-trabalho condicional e não-condicional separando os domicílios de acordo com o número de adultos. Segundo GSW (2004, p. 15), isto permite identificarmos "em quais tipos de domicilios bá uma redução da polarização quando esta é realizada condicional nas características dos indivíduos e em que tipo de domicilios a polarização permanece inexplicada." Assim, no Apêndice, repetimos o exercício da Tabela 6 para domicílios com 1, 2, 3 e 4 ou mais adultos separadamente. Quando nos concentramos apenas nos domicílios com um adulto, novamente a polarização - condicional ou não - é negativa, havendo tendência de elevação entre 1981 e 2003. Ao analisarmos as taxas de não-trabalho condicionais, apenas a variação por sexo e de todas as variáveis combinadas mostram diferença (na verdade, são maiores) em relação à taxa não-condicional.

Já quando consideramos apenas os domicílios com dois adultos, a variação por sexo reduz expressivamente a taxa domiciliar de não-trabalho prevista em 1981, e menos em 2003, relativamente à taxa não-condicional. Assim, é neste tipo de família que se repete o padrão por sexo verificado para a análise agregada. Ou seja, com o tempo, o padrão de trabalho dos cônjuges se assemelhou, fazendo com que a desagregação por sexo diferisse menos da taxa de não-trabalho não-condicional.

$\mathrm{Na}$ análise com três adultos em 1981, as taxas previstas condicionais para sexo e todas as variáveis conjuntamente são bem menores que as não-condicionais. Já em 2003, apenas estas duas decomposiçôes, mais a de região, mostram polarização positiva. Assim, região, idade e educação se tornaram mais importantes para explicar a polarização em 2003 do que em 1981. Por fim, na análise com 4 ou mais adultos, há muito pouca variação, sendo que não há garantia de que as variações que ocorrem são estatisticamente significativas.

Uma questão bastante importante para avaliarmos as consequiências da polarização é o fato de haver ou não crianças presentes nos domicílios em que ninguém trabalha. Obviamente, a probabilidade de uma criança ter seu desenvolvimento prejudicado, ou ainda de ter que trabalhar, é maior se ela vive em um domicílio em que nenhum adulto trabalha. Deste modo, apresentamos na Tabela 7 as medidas de fração de do- 
micílios sem trabalho e de polarização desagregadas por número de adultos e pela presença ou não de crianças nos domicílios. ${ }^{7}$

Com relação à proporção de cada tipo de arranjo domiciliar, a Tabela 7 mostra que apesar de representar a maior proporção de famílias, as com dois adultos e crianças apresentaram redução em 2003. Por outro lado, houve aumento na proporção de domicílios com um adulto, com e sem crianças e de dois adultos sem crianças. Por fim, houve redução na fração de domicílios com 3 e 4 adultos com crianças. Essas tendências refletem a redução da taxa de natalidade no Brasil, bem como o aumento do número de separações, elevando o número de crianças morando apenas com o pai, a mãe ou outro parente. Já o crescimento na parcela de domicílios com mais de dois adultos e sem crianças está ligado ao fato de que, no Brasil, as pessoas estão se casando cada vez mais tarde.

TABELA 7 - TAXA DE NÃO-TRABALHO DOMICILIAR E POLARIZAÇÃO POR NÚMERO DE ADULTOS E PRESENÇA DE CRIANÇA

\begin{tabular}{|c|c|c|c|c|c|c|c|c|}
\hline \multirow[b]{2}{*}{ Criança } & \multicolumn{2}{|c|}{1 adulto } & \multicolumn{2}{|c|}{2 adultos } & \multicolumn{2}{|c|}{3 adultos } & \multicolumn{2}{|c|}{$4+$ adultos } \\
\hline & Não & Sim & Não & Sim & Não & Sim & Não & Sim \\
\hline \multicolumn{9}{|c|}{ Fração dos Domicílios } \\
\hline 1981 & 5,5 & 3,3 & 11,3 & 47,1 & 5,3 & 11,5 & 5,3 & 10,7 \\
\hline 2003 & 8,5 & 5,3 & 15,9 & 40,1 & 8,4 & 8,9 & 6,7 & 6,0 \\
\hline \multicolumn{9}{|c|}{ Taxa de não-trabalho domiciliar } \\
\hline 1981 & 10,8 & 16,4 & 8,9 & 3,9 & 6,0 & 3,3 & 1,9 & 1,2 \\
\hline 2003 & 12,6 & 19,9 & 9,2 & 5,2 & 5,2 & 4,5 & 2,7 & 2,3 \\
\hline \multicolumn{9}{|c|}{ Polarização não-condicional } \\
\hline 1981 & $-27,2$ & $-21,6$ & $-5,5$ & $-10,5$ & 0,6 & $-2,2$ & 0,4 & $-0,2$ \\
\hline 2003 & $-21,4$ & $-14,2$ & $-2,4$ & $-6,4$ & 1,2 & 0,6 & 1,7 & 1,3 \\
\hline \multicolumn{9}{|c|}{ Polarização condicional } \\
\hline 1981 & $-21,2$ & $-37,4$ & $-3,1$ & $-4,2$ & 0,5 & $-1,3$ & 0,6 & $-0,1$ \\
\hline 2003 & $-17,0$ & $-23,7$ & $-2,0$ & $-4,2$ & 0,9 & $-0,1$ & 1,6 & 0,8 \\
\hline
\end{tabular}

Fonte: Elaboração própria com base nos dados da PNAD.

Já a análise da proporção de domicílios sem trabalho revela diferenças expressivas de acordo com a presença de crianças, além da maior incidência do não-trabalho quanto menor o número de adultos do domicílio. Assim, entre os domicílios com um adulto, a taxa de não-trabalho naqueles com crianças é maior que nos sem crianças. Assim, crianças que moram com apenas um adulto têm maiores probabilidades de pertencerem a um domicílio sem trabalho adulto e, portanto, estariam mais susceptíveis ao trabalho infantil. Entretanto, analisando os domicílios com dois adultos, este resul-

7 Consideramos como crianças todos os indivíduos de menos de 14 anos do domicílio, exceto pensionistas, empregados domésticos e parentes de empregados domésticos. 
tado se inverte, havendo menos domicílios com crianças presentes e sem trabalho do que entre os sem crianças, resultado também verificado por GSW (2004), exceto para a Austrália.

Cabe salientar que a proporção de domicílios sem trabalho entre aqueles com um adulto é bem menor no Brasil do que nos países estudados por GSW (2004). Vale ressaltar o caso dos Estados Unidos, que nas famílias com um adulto e sem crianças havia taxas de não-trabalho mais próximas às encontradas para o Brasil. Em 2000, nos domicílios com um adulto e crianças, a proporção daqueles sem trabalho era de $22,7 \%$, embora maior que a taxa brasileira de 19,9\% em 2003. Segundo GSW (2004), a forte redução desta proporção nos EUA ocorreu após o fim do AFDC em 1996. Além disso, vale notar que no Brasil, assim como nos países estudados por GSW (2004), as maiores taxas de não-trabalho ocorrem nos domicílios com um adulto e crianças presentes, exceto a Espanha em 2000.

Analisando a evolução entre 1981 e 2003, houve aumentos na proporção de domicílios sem trabalho entre aqueles com qualquer número de adultos com crianças presentes, $\mathrm{o}$ que sempre representa uma preocupação, apesar do aumento não ter sido tão grande, exceto nos com apenas um adulto.

No que diz respeito às medidas de polarização, houve crescimento na medida nãocondicional em todas as categorias. Porém, a categoria com menor polarização era a dos domicílios com um adulto sem crianças presentes, com polarização fortemente negativa, resultado significativamente diferente do encontrado por GSW (2004), em que a polarização neste tipo de domicílio era bem maior que nos demais no caso da Grã-Bretanha e da Austrália.

No que diz respeito à polarização condicional, a proporção de domicílios sem trabalho com um adulto e crianças era muito menor em 1981 (16,4\%) do que seria de se esperar se o não-trabalho fosse distribuído uniformemente, mesmo permitindo a variação por educação, sexo, região e idade $(53,8 \%)$. Entretanto, há expressivo aumento da polarização neste grupo em 2003 (-23,7\%), não deixando, no entanto, de ser a mais baixa entre todos os grupos. É importante perceber, contudo, que este aumento se deu pela diminuição na probabilidade condicional de não-trabalho dentro destes domicílios, que caiu para 43,6\% em 2003.

\section{CONCLUSÕES}

O presente trabalho procurou analisar a questão da polarização do trabalho no Brasil. A análise mostrou que no Brasil a polarização do trabalho é menor do que em países 
como a Grã-Bretanha, Alemanha, Austrália e Estados Unidos. Ou seja, há menos domicílios sem trabalho no Brasil do que seria de se esperar caso o trabalho fosse distribuído aleatoriamente na população. Entre os fatores que podem explicar este resultado merece destaque a ausência de uma rede eficaz de proteção social que permita aos domicílios empreender um processo de busca mais selecionada de trabalho.

Além disso, concluímos que a alteração na composição domiciliar (em termos de número de adultos) pouco contribuiu para o aumento da polarização. Por outro lado, cerca de $85 \%$ do aumento da polarização ocorreu dentro de domicílios com dois adultos. Assim, o aumento da proporção de domicílios em que os dois adultos presentes não trabalham é uma questão importante a ser melhor investigada para se descobrir o que está gerando esta situação.

Um resultado importante é que em cerca de $20 \%$ dos domicílios com um adulto e com crianças presentes o adulto estava sem trabalho em 2003. Uma possível causa deste resultado seria o baixo acesso a creches por parte de mães solteiras, o que dificultaria a entrada das mães no mercado de trabalho. Entretanto, uma continuidade deste trabalho é necessária para avaliarmos em que medida isto está realmente ocorrendo.

Neste trabalho, tratamos desempregados e inativos da mesma maneira. Uma extensão natural seria procurar estudar a polarização do desemprego e da inatividade, em que estes fenômenos seriam considerados separadamente.

\section{REFEREANCIAS BIBLIOGRÁFICAS}

Avelino, R.; Menezes-Filho, N. Estimação da oferta de trabalho das mulheres no Brasil. Estudos Econômicos, v. 33, n. 4, p. 639-664, out./dez. 2003.

Chahad, J.; Picchetti, P. A evolução da taxa de desemprego estrutural no Brasil: uma análise entre regiões e características dos trabalhadores. Anais do XXXI Encontro Nacional de Economia - ANPEC, 2003.

Dawkins, P.; Gregg, P.; Scutella, R. Employment polarization in Australia. CMPO Working Paper Series, n. 02/50, 2002.

Fernandes, R.; Felício, F. The entry of the wife into the labor force in response to the husband's unemployment: a study of the added worker effect in Brazilian metropolitan areas. Economic Development and Cultural Change, v. 53, p. 887911, 2005.

Gonzaga, G; Reis, M. Os efeitos trabalhador adicional e desalento no Brasil. Anais do XXXIII Encontro Nacional de Economia - ANPEC, 2005.

Gregg, P.; Scutella, R.; Wadsworth, J. Reconciling workless measures at the individual and household level: theory and evidence from the United States, Britain, 
Spain, Germany and Australia. Centre for Economic Performance Working Paper, n. $635,2004$.

Gregg, P.; Wadsworth, J. Why we should (also) measure worklessness at the household level. Theory and evidence from Britain, Spain, Germany and the United States. Centre for Economic Performance Working Paper, n. 1168, 2002.

Everything you ever wanted to know about measuring worklessness and polarization at the household level but were afraid to ask. Oxford Bulletin of Economics and Statistics, v. 63, special issue, p. 777-806, 2001.

Guerra, V. S. Principais mudanças introduzidas nos conceitos e definiçôes da PNAD para as pesquisas de $1992 \mathrm{em}$ diante. 1997. Mimeografado.

Kassouf, A. Aspectos sócio-econômicos do trabalbo infantil no Brasil. Brasília: Ministério da Justiça, 2002. 124p.

Picchetti, P.; Menezes-Filho, N. A. Desemprego. In: Lisboa, M. B.; Menezes-Filho, N. A. (orgs.), Microeconomia e sociedade no Brasil. Fundação Getúlio Vargas, 2001.

- Os determinantes da duração do desemprego em São Paulo. Pesquisa e Planejamento Econômico, v. 30, n. 1, 2000.

Picchetti, P.; Fernandes, R. Uma análise da estrutura do desemprego e da inatividade no Brasil metropolitano. Pesquisa e Planejamento Econômico, v. 29, n. 1, 1999.

Ramos, L. O funcionamento do mercado de trabalho metropolitano brasileiro no período 1991-2001: Texto de Discussão. Rio de Janeiro: IPEA, nov. 2000.

Scorzafave, L.; Menezes-Filho, N. Participação feminina no mercado de trabalho brasileiro: evolução e determinantes. Pesquisa e Planejamento Econômico, v. 31, n. 3, p. 44l-477, 2001. 


\section{APENNDICE}

TABELA AI - TAXAS DE NÃO-TRABALHO CONDICIONAIS PARA DOMICÍLIOS COM I ADULTO - 1981-2003

\begin{tabular}{lccc}
\hline & 1981 & 2003 & Variação \\
\hline Taxa domiciliar de não-trabalho observada $(\mathrm{w})$ & 12,9 & 15,4 & 2,5 \\
Taxa domiciliar de não-trabalho prevista não-condicional $(\hat{\mathrm{w}})$ & 38,0 & 34,1 & $-3,9$ \\
\hline Taxa domiciliar de não-trabalho prevista condicional $\left(\hat{\mathrm{W}}_{\mathrm{k}}\right)$ & & & \\
$\quad$ Sexo & 40,0 & 36,0 & $-4,0$ \\
Região & 38,1 & 34,0 & $-4,1$ \\
$\quad$ Idade & 38,0 & 33,1 & $-4,9$ \\
Educação & 38,1 & 34,2 & $-3,9$ \\
Sexo, Região, Idade e Educação combinados & 40,1 & 35,0 & $-5,1$ \\
\hline
\end{tabular}

Fonte: Elaboração própria com base nos dados da PNAD.

TABELA A2 - TAXAS DE NÃO-TRABALHO CONDICIONAIS PARA DOMICÍLIOS COM DOIS ADULTOS - 1981-2003

\begin{tabular}{lrrc}
\hline & 1981 & 2003 & Variação \\
\hline Taxa domiciliar de não-trabalho observada $(\mathrm{w})$ & 4,9 & 6,4 & 1,5 \\
Taxa domiciliar de não-trabalho prevista não-condicional $(\hat{\mathrm{w}})$ & 14,4 & 11,6 & $-2,8$ \\
\hline Taxa domiciliar de não-trabalho prevista condicional $\left(\hat{\mathrm{w}}_{\mathrm{k}}\right)$ & & & \\
$\quad$ Sexo & 9,8 & 10,4 & 0,6 \\
Região & 14,8 & 12,0 & $-2,8$ \\
Idade & 14,1 & 11,3 & $-2,8$ \\
Educação & 15,1 & 12,4 & $-2,7$ \\
Sexo, Região, Idade e Educação combinados & 8,9 & 9,9 & 1,0 \\
\hline
\end{tabular}

Fonte: Elaboração própria com base nos dados da PNAD.

TABELA A3 - TAXAS DE NÃO-TRABALHO CONDICIONAIS PARA DOMICÍLIOS COM 3 ADULTOS - 1981-2003

\begin{tabular}{lccc}
\hline & 1981 & 2003 & Variação \\
\hline Taxa domiciliar de não-trabalho observada $(\mathrm{w})$ & 4,1 & 4,8 & 0,7 \\
Taxa domiciliar de não-trabalho prevista não-condicional $(\hat{\mathrm{w}})$ & 5,5 & 4,0 & $-1,5$ \\
\hline Taxa domiciliar de não-trabalho prevista condicional $\left(\hat{\mathrm{w}}_{\mathrm{k}}\right)$ & & & \\
$\quad$ Sexo & 4,9 & 4,2 & $-0,7$ \\
Região & 7,0 & 4,9 & $-2,1$ \\
Idade & 7,2 & 5,0 & $-2,2$ \\
Educação & 7,3 & 5,0 & $-2,3$ \\
Sexo, Região, Idade e Educação combinados & 4,9 & 4,4 & $-0,5$ \\
\hline
\end{tabular}

Fonte: Elaboração própria com base nos dados da PNAD. 
TABELA A4 - TAXAS DE NÃO-TRABALHO CONDICIONAIS PARA DOMICÍLIOS COM 4 OU + ADULTOS - 1981-2003

\begin{tabular}{lccc}
\hline & 1981 & 2003 & Variação \\
\hline Taxa domiciliar de não-trabalho observada $(\mathrm{w})$ & 1,4 & 2,5 & 1,1 \\
Taxa domiciliar de não-trabalho prevista não-condicional $(\hat{\mathrm{w}})$ & 1,4 & 1,0 & $-0,4$ \\
\hline Taxa domiciliar de não-trabalho prevista condicional $\left(\hat{\mathrm{w}}_{\mathrm{k}}\right)$ & & & \\
$\quad$ Sexo & 1,2 & 1,1 & $-0,1$ \\
Região & 2,0 & 1,4 & $-0,6$ \\
Idade & 2,2 & 1,6 & $-0,6$ \\
Educação & 2,0 & 1,3 & $-0,7$ \\
Sexo, Região, Idade e Educação combinados & 1,3 & 1,3 & 0,0 \\
\hline
\end{tabular}

Fonte: Elaboração própria com base nos dados da PNAD. 